\title{
Intelligent Systems and Its Applications in Robotics
}

\author{
Okyay Kaynak \\ UNESCO Chair on Mechatronics, Boğaziçi Üniversitesi, Bebek, 34342 Istanbul, Turkey \\ okyay.kaynak@boun.edu.tr
}

\begin{abstract}
The last decade of the last millennium is characterized by what might be called the intelligent systems revolution, as a result of which, it is now possible to have man made systems that exhibit ability to reason, learn from experience and make rational decisions without human intervention. Prof. Zadeh has coined the word MIQ (machine intelligence quotient) to describe a measure of intelligence of man-made systems. In this perspective, an intelligent system can be defined as a system that has a high MIQ.

The presentation dwells upon how to define "intelligence" of man-made machines and continues with a discussion on computational intelligence, its main components and compares it with biological and artificial intelligence. The paradigm shift from computation with measurements to computation with perceptions is also pointed out to. The state-of-art reached in intelligent systems is overviewed with examples and a perspective on the future is given, based on "futurology" rather than "prophecy". The reasons behind the slow pace of developments are discussed, tying it to the availability of the computing power. The trends seen in this respect over the last century are overviewed and it is argued that the Moore's Law will have to reach an end, not so much because of technological difficulties but because of financial ones. Quantum and molecular computing are offered as possible alternatives. The talk closes with a consideration of the possible research directions in mechatronics and robotics as driving forces behind the development of intelligent systems.
\end{abstract}

